\title{
Combination interaction of Taylor-Goertler vortices in curved shear layer of supersonic jet
}

\author{
V. Zapryagaev*, V. Pickalov, N. Kiselev, A. Nepomnyashchiy \\ Institute of Theoretical \& Applied Mechanics, Russian Academy \\ of Sciences, Siberian Branch, Institutskay str. 4/1, Novosibirsk, 630090, RUSSIA \\ e-mail: zapr@itam.nsc.ru - Web page: http://www.itam.nsc.ru/
}

The importance of the study of the turbulent mixing processes in a shear layer of the supersonic jet is caused by an extensive use of jets in practice. The brief review of experimental research of an origin formation and development of streamwise vortex structures in a mixing layer of a supersonic jet is presented. It has been experimentally established that the initial section of the shear layer of a supersonic nonisobaric jet exhausting from an axisymmetric nozzle has a three-dimensional structure. The presence of these vortex structures is interpreted as a three-dimensional structure of the turbulent flow [1,2]. Main goal of this paper consist in a finding of the conditions for control of the process of the generation streamwise vortex structures in the supersonic jet shear layer. Results of measurement of distribution of Pitot pressure in supersonic nonisobaric jet both in radial and in azimuthal directions are submitted. Azimuthal heterogeneity, corresponding to streamwise vortices in a shear layer, is analyzed with use as Fourier analysis and with application of wavelet analysis. Influence of the shear layer curvature on development process of streamwise vortices is discussed in [3]. For more carefully investigation of this question it was selected two jets with identical gasdynamic parameters but with different curvatures in initial section of the jet shear layer.

$\mathrm{Ma}=1.0$
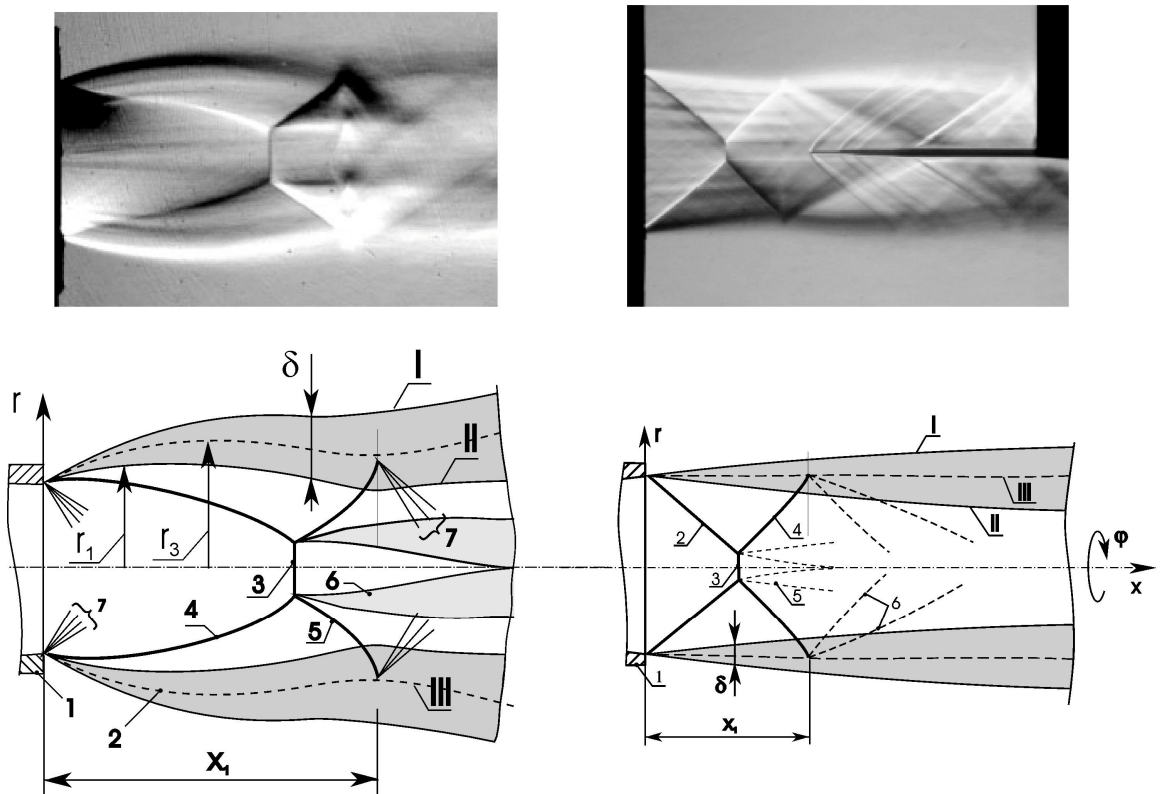

Fig. 1.

Supersonic jets were issued from the convergent nozzle $(\mathrm{Ma}=1.0)$ and convergent-divergent Laval nozzle $(\mathrm{Ma}=2.0)$. Both nozzles were manufactured with polished inner surfaces. Jets were issued to the embient ambient air. The radius of the nozzle exit was equal $R_{a}=15 \mathrm{~mm}$. The experiments have been performed in the special jet insert for hypersonic aerodynamic wind tunnel T-326 of ITAM SB RAS with cold air. Experimental data were obtained for nozzle pressure ratio $\mathrm{Npr}=\mathrm{Po} / \mathrm{Pc}=5.0$ (here Po is stagnation pressure, Pc - ambient pressure in test chamber). Results of the turbulent flow visualization and jets schemes are presented in Fig.1. 
On left scheme for convergent nozzle $(\mathrm{Ma}=1)$ it is shown the structure of the underexpanded jet with $\mathrm{n}_{\mathrm{p}}=2.64\left(\mathrm{n}_{\mathrm{p}}=\mathrm{Pa} / \mathrm{Pc}, \mathrm{Pa}\right.$ is pressure on nozzle exit). Here I - outside jet boundary; II- internal shear layer boundary; III- shear layer centerline, $\mathrm{x}, \mathrm{r}$, and $\varphi$ are the cylindrical coordinates; 1- nozzle; 2 shear layer ( $\delta$ is thickness of shear layer); 3- Mach disk; 4 and 5 - barrel and reflected compression shocks; 6- shear layer behind the triple point; 7- expansion fan.

On right scheme for Laval nozzle $(\mathrm{Ma}=2)$ it is shown the structure of the overexpanded jet with $\mathrm{n}_{\mathrm{p}}=0.64$. Here 1- nozzle; 2- compression shock; 3- Mach disk; 4 - reflected shock; 5 - shear layer behind the triple point; 6 - expansion fan, $\mathrm{x}_{1}$ - length of first jet cell.

Experiments have been performed as for polished inner nozzle surface as for artificial microroughness lied on inner nozzle surface with equal step azimuthal angle 45 degrees. Height of microroughness is equal $\mathrm{k}=0.04 \mathrm{~mm}$. Single microroughness is form of triangles and similar form used in work [2]. The measurements in the shear layer of the jet were performed for five cross sections in initial jet region. Pitot pressure probe with outer diameter equal $0.6 \mathrm{~mm}$ (right photo in Fig.1) was used to obtain of radial and azimuthal pressure distributions in the jets.

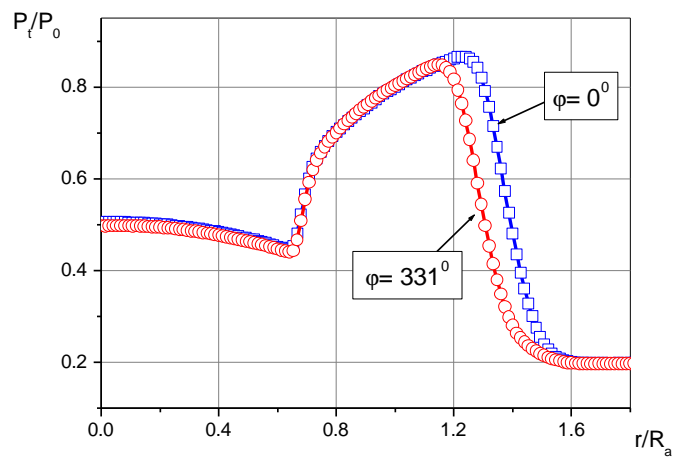

Fig.2.

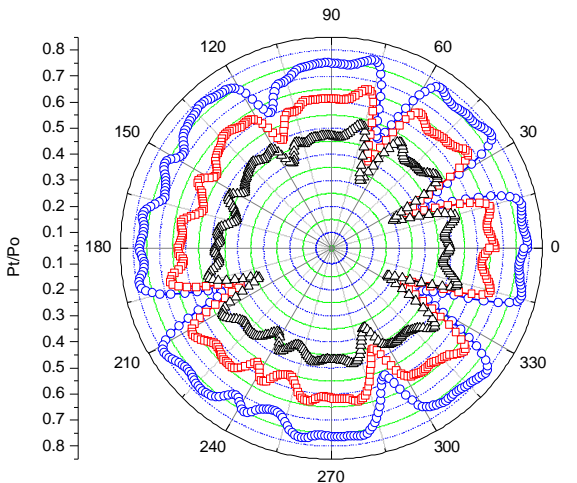

Fig.3.

The radial Pitot pressure distributions for two azimuthal angles ( $\varphi=0$ and 331 degrees) for $\mathrm{x} / \mathrm{Ra}=2.0$ $(\mathrm{Ma}=1)$ and for artificial microroughness are presented in Fig.2. We can see essential difference between two radial profiles. For example, azimuthal angle dependences $\mathrm{P}_{\mathrm{t}}(\varphi)$ measured in the crosssections $\mathrm{x} / \mathrm{Ra}=2.0(\mathrm{Ma}=1)$ for three radial coordinates are shown in Fig.3 $(\mathrm{r} / \mathrm{Ra}=1.3 ; 1.33$ and 1.36). Azimuthal distributions of pressure display distinct maxima and minima at one and the same angles $\varphi$, which observation is indicative of the stationary character of the streamwise vortices in the jet shear layer, proving simultaneously good reproducibility of detected azimuthal disturbances. Locations of Pitot pressure minima are corresponded to positions of artificial microroughnesses.

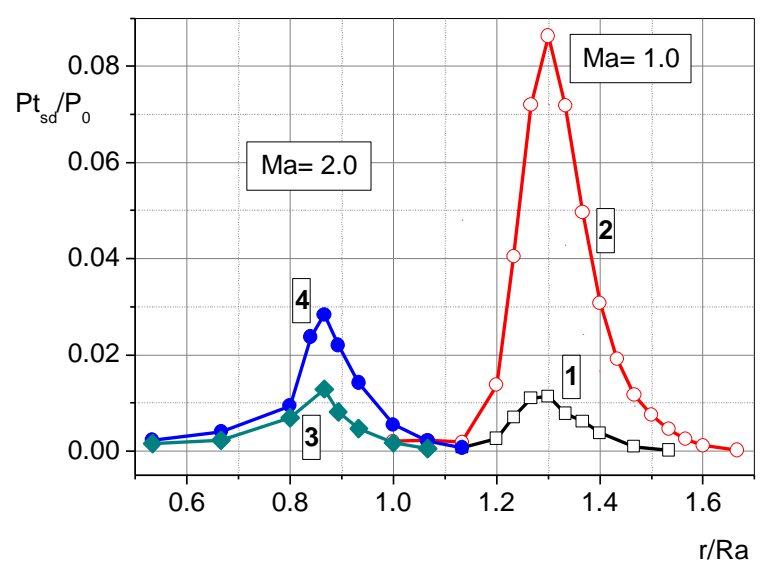

Fig.4. 
The root-mean-square amplitude $\mathrm{P}_{\mathrm{sd}} / \mathrm{P}_{\mathrm{o}}$ of pressure variations was calculated for each measurement azimuthal dependence on the normalized Pitot pressure for different radius. In Fig.4 four curves are presented. Data are corresponded to $\mathrm{x} / \mathrm{R}_{\mathrm{a}}=2.0$. Curves 1,2 are obtained for underexpended jet $(\mathrm{Ma}=1)$, curves 3, 4 are obtained for overerexpended jet $(\mathrm{Ma}=2)$. Curves 1,3 are corresponded to small natural microroughnesses $(\mathrm{k}=0.0025 \mathrm{~mm})$ and curves 2,4 are corresponded to artificial microroughnesses $(\mathrm{k}=0.04 \mathrm{~mm})$. We can see similar dependences $\mathrm{P}_{\mathrm{sd}}(\mathrm{r})$ for small artificial microroughness (curves 1 and 3) - maximum $\left\{\mathrm{P}_{\mathrm{sd}} / \mathrm{Po}\left(\mathrm{r} / \mathrm{R}_{\mathrm{a}}\right\} \sim 0.01\right)$. For artificial microroughnesses (curves 2 and 4) we can see essential difference, which may be explained by influence of the streamline curvature in the shear layer.
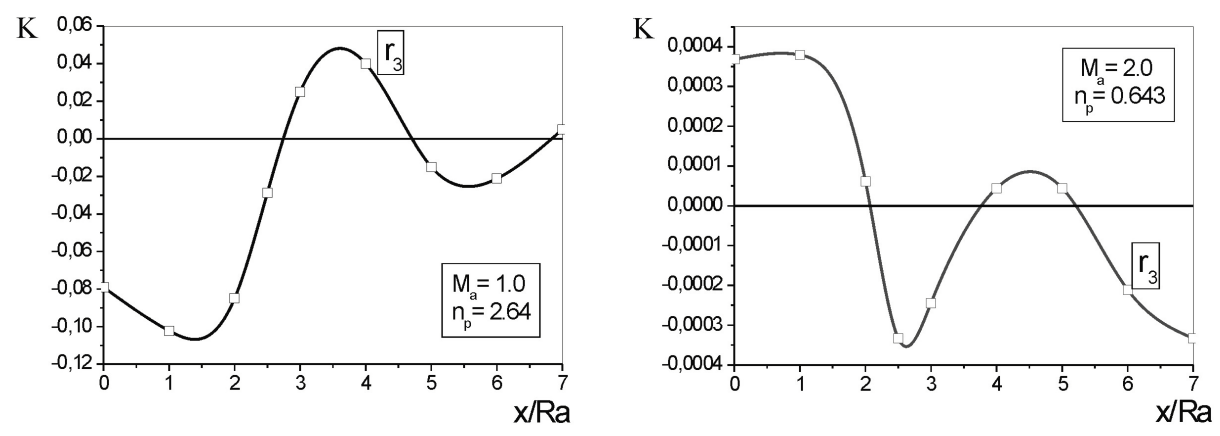

Fig.5.

Streamline curvature $\mathrm{Cr}$ in the shear layer was calculated as for underexpanded and for overexppanded jets by using radial profiles for different jet cross sections. In fig. 5 dimensionless curvature $\mathrm{K}=\mathrm{Cr} / \mathrm{R}_{\mathrm{a}}$ is presented in dependence of axial distance $\mathrm{x} / \mathrm{R}_{\mathrm{a}}$ for both jet regimes. We can see large negative value of a dimensionless curvature $\mathrm{K} \sim-0.11$ for $\mathrm{x} / \mathrm{R}_{\mathrm{a}}=1.5$ and for middle of the shear layer $\left(r_{3}\right)$ for underexpanded jet. For overexpanded jet a dimensionless curvature is very little positive value $\mathrm{K} \sim+0.0004$. Amplification of the azimuthal disturbances would be observed namely for negative streamline curvature simultaneously with negative derivation of velocity on radial direction according to Rayleigh criteria [4] for Goertler flow instability. These conditions are in the shear layer for an underexpanded jet.

A spectral analysis of stationary azimuthal disturbances in the shear layer of a supersonic jet was performed by using of the Fourier series. It was observed that the Fourier amplitudes of streamwise vortices for natural and artificial conditions are significantly different. In Fig.6 the spectrum of Fourier-amplitudes corresponding to the azimuthal Pitot pressure distribution which is registered in the middle of a shear layer at radius $r / R_{a}=1.3$ is shown (the spectra corresponds to underexpanded jet $\mathrm{Ma}=1$ with artificial microroughnesses on the nozzle surface). The ordered law in values of azimuthal wave number $\mathrm{n}$ which are registered the maximal values of spectral amplitudes is observed. Sequences of values of azimuthal number $\mathrm{n}$ it is defined by the basic fashion (a step artificial microroughnesses $\left.45^{\circ}\right) \mathrm{n}_{0}=360^{\circ} / 45^{\circ}=8$ and additional azimuthal wave number $\mathrm{n}_{1}=3$. This law in behavior of peaks of spectral amplitudes is also observed and at other jet radii. The most probable explanation of the found law in spectra of Fourier amplitudes of stationary streamwise vortices should be counted three-wave resonant interaction. Analog phenomena was investigated by Craik [5] for disturbances in boundary layer.

Continuous wavelet transformation of a signal using well-known MHAT-wavelet named by «Mexican hat » has been applied also for the analysis of the measured azimuthal distribution of Pitot pressure in jet. Wavelet coefficients of one-dimensional wavelet transform are shown in Fig.7. The axis of abscissas corresponds to an azimuthal angle, an axis of ordinate corresponds to an axis of logarithmic wavelet scales. Numerous periodic details are visible in the bottom part of the waveletimage. Eight "arches" are defined by number of artificial microroughnesses. Results of the wavelet analysis point out on the coherent structures in a shear layer that correlates with the Fourier analysis results. 


\section{$\log _{2}(2 \pi / a)$}

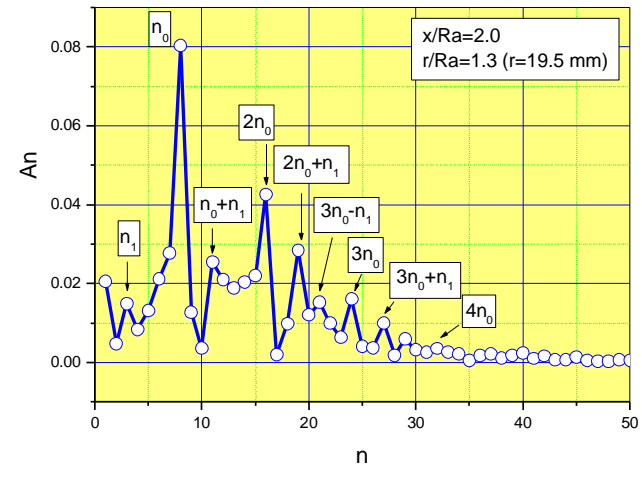

Fig.6.

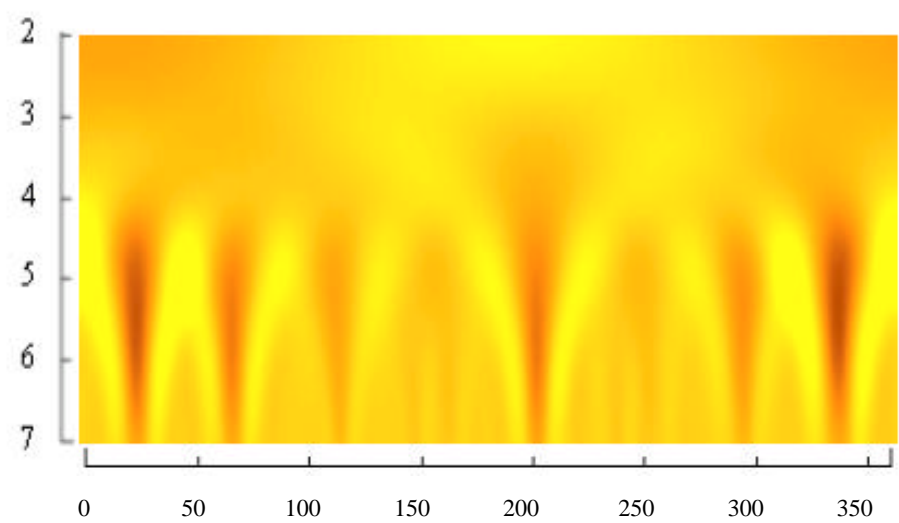

Fig.7.

In conclusion it is noted that comparison of Pitot pressure azimuthal variations in shear layers for two supersonic jets was performed. Jets issued in an ambient space from as convergent $(\mathrm{Ma}=1)$ as convergent-divergent Laval $(M=2)$ nozzles with same nozzle pressure ratio and same jet Mach number. Mean squared values of azimuthal Pitot pressure variations in jet shear layers are approximately constant for both jets for small microroughness on the inner nozzle surface $(\mathrm{k} 2.5 \mathrm{mkm})$. Experimental mean squared values of azimuthal Pitot pressure variations in jet shear layers are essentially different for large artificially microroughness on the inner nozzle surface $(\mathrm{k}=40.0 \mathrm{mkm})$ for those jets because shear layer curvature is different for overexpanded and underexpanded jets. Negative curvature is favour condition for Goertler flow instability growth in jet shear layer according to Rayleigh criteria. Fourier spectra analysis results of azimuthal Pitot pressure variations for underexpanded jet with artificial microroughness on the nozzle surface point to presence of the combination nonlinear interaction for different azimuthal modes. Those azimuthal modes correspond to streamwise Taylor-Goertler vortices with different scales. Wavelet analysis results gave additional information and are in good correspondence to Fourier analysis.

This work was supported by grant INTAS No. 99-0785 and grant RFBR No.02-01-00515.

\section{REFERENCES}

[1] V.I. Zapryagaev, A.V. Solotchin. Spatial structure of flow in the initial section of a supersonic underexpanded jet, Preprint No.23-88, Academy of Sciences USSR, Siberian Division, ITAM, Novosibirsk, 1988 (in Russian).

[2] V.I. Zapryagaev. A method of mixing process control in a shear layer of a supersonic nonisobaric jet, IUTAM Symposium on Mechanics of Passive and Active Flow Control, Eds. G.E.A. Meier and P.R.Viswanath, Kluwer Academic Publishers, 1999.

[3] A. Krothapalli, P.J. Strykowski, C.J. King. Origin of streamwise vortices in supersonic jets, AIAA J., 36(5), 1999.

[4] W.S. Saric. Goertler vortices, Annual Rev. Fluid Mech. 26, 379-409, 1994

[5] A.D.D. Craik. Non-linear resonant instability in boundary layers, J.Fluid.Mech., 50(2), 1971. 